\title{
Latest highlights from CDF
}

\author{
Massimo Casarsa* \\ (on behalf of the CDF Collaboration) \\ Fermi National Accelerator Laboratory, Batavia, IL, USA \\ E-mail: casarsalfnal.gov
}

The CDF II experiment at the Fermilab Tevatron collider has been running steadily for many years accumulating large datasets which allow to probe many facets of the Standard Model and its possible extensions over a wide range of processes. This contribution presents an overview of the CDF physics program focusing on the most recent results as of April 2010.

XVIII International Workshop on Deep-Inelastic Scattering and Related Subjects, DIS 2010 April 19-23, 2010

Firenze, Italy

\footnotetext{
*Speaker.
} 


\section{Introduction}

The CDF II experiment at the Fermilab Tevatron collider has been steadily acquiring data since the beginning of 2002. The large accumulated datasets allow CDF to pursue a very rich and broad physics program, which is probing many facets of the Standard Model (SM) and its possible extensions over a wide range of processes, whose cross sections span over 10 orders of magnitude, from the tens of millibarns of the total $p \bar{p}$ cross section to the fraction of a picobarn expected for the production of the SM Higgs boson.

The CDF investigation of the fundamental constituents of matter is organized on the basis of the phenomena and the sector of the Standard Model which are being explored: the study of the strong interactions between the quarks and gluons in the colliding nucleons, the $b$ - and $c$-quark flavor physics, the properties of the top quark and the electroweak gauge bosons, the electroweak symmetry breaking and the Higgs boson, the searches for physics beyond the Standard Model. Following this baseline the contribution will review the CDF physics program focusing on the most recent results as of April 2010: Sec. 2 briefly describes the experimental apparatus, the Tevatron collider and the CDF detector, in the subsequent Sections the results are presented.

\section{The experimental apparatus}

The Tevatron is a $1-\mathrm{km}$ radius proton-antiproton superconducting synchrotron that operates at a center of mass energy of $1.96 \mathrm{TeV}$. 36-bunch beams of protons and antiprotons collide at two interaction points, where the $\mathrm{CDF}$ and $\mathrm{D} \emptyset$ detectors are located, with an instantaneous luminosity in the range $0.8-4.0 \times 10^{32} \mathrm{~cm}^{-2} \mathrm{~s}^{-1}$. Typically the Tevatron delivers to each experiment an integrated luminosity of $60-70 \mathrm{pb}^{-1}$ per week for a total delivered luminosity in Run II of $9 \mathrm{fb}^{-1}$.

CDF is a multipurpose detector with a cylindrical symmetry. The detector geometry is described by the azimuthal angle $\varphi$ and the pseudo-rapidity $\eta=-\log (\tan \vartheta / 2)$, where $\vartheta$ is the polar angle of a particle with respect to the proton beam axis (positive $z$-axis). The pseudo-rapidity of a particle originating from the center of the detector is referred to as $\eta_{\text {det }}$. The most relevant components of the CDF II detector are briefly outlined here; a more complete description can be found elsewhere [1]. The trajectories of charged particles are reconstructed using silicon microstrip detectors and a 96-layer open cell drift chamber embedded in a $1.4 \mathrm{~T}$ solenoidal magnetic field. For $\left|\eta_{\text {det }}\right| \leq 1$, a particle traverses all 96 layers of the drift chamber; this decreases to zero at $\left|\eta_{\text {det }}\right| \approx 2$. The silicon system provides coverage with 6 (7) layers with radii between $2.4 \mathrm{~cm}$ and $28 \mathrm{~cm}$ for $\left|\eta_{\text {det }}\right|<1\left(1<\left|\eta_{\text {det }}\right|<2\right)$. Outside of the solenoid are electromagnetic and hadronic sampling calorimeters segmented in a projective tower geometry. The first hadronic interaction length $\lambda$ of the calorimeter, corresponding to 19-21 radiation lengths $X_{0}$, uses lead absorber for measuring the electromagnetic component of showers, while the section extending to 4.5-7 $\lambda$ uses iron to contain the hadronic component. The calorimeters are divided in a central $\left(\left|\eta_{\operatorname{det}}\right|<1\right)$ and forward $\left(1.1<\left|\eta_{\text {det }}\right|<3.6\right)$ region. Shower maximum detectors embedded in the electromagnetic calorimeters at approximately $6 X_{0}$ help in the position measurement and background suppression for electrons. Outside of the central calorimeters are scintillators and four layers of drift chambers for reconstructing and identifying muons up to $\left|\eta_{\text {det }}\right|<1.5$. 


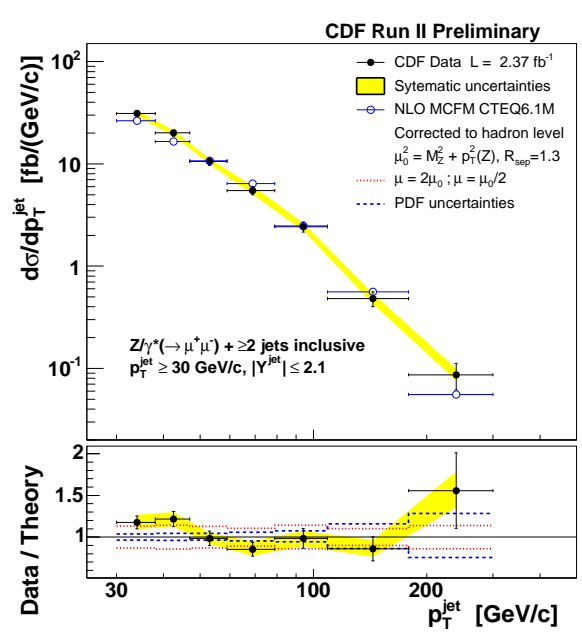

Figure 1: Measured inclusive jet differential cross section (black dots) as a function of $p_{T}^{\text {jet }}$ in $Z / \gamma^{*}+\geq 2$ jets events compared to NLO pQCD predictions (open circles).

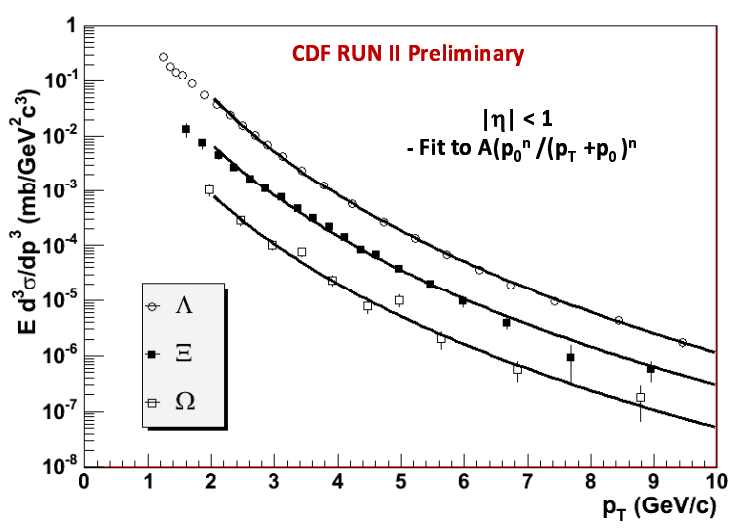

Figure 2: Inclusive differential cross sections as a function of the transverse momentum for centrally produced $\Lambda^{0}, \Xi^{ \pm}$and $\Omega^{ \pm}$. The fits to the data are superimposed.

\section{Overview of recent CDF results}

In the following Sections a selection of the most recent results from the CDF Collaboration will be presented: quantum chromodynamics (QCD) results in Sec. 3.1, bottom and top quark physics in Secs. 3.2 and 3.3, studies on the electroweak gauge bosons in Sec. 3.4, searches for a SM Higgs in Sec. 3.5 and searches for phenomena beyond the Standard Model in Sec. 3.6

\subsection{QCD results}

The study of the partons which partake in the strong interactions provides stringent tests of the perturbative QCD [2] (pQCD) predictions and gives insights into the structure of the proton and the antiproton. Moreover, a precise measurement of the cross sections of the QCD processes is fundamental to understand the backgrounds for Standard Model measurements and new physics searches.

A test of the $\mathrm{pQCD}$ predictions is given by the CDF measurement of the inclusive production of collimated jets of hadrons in association with a $Z / \gamma^{*}$ boson. The $Z / \gamma^{*}$ bosons are reconstructed in the final state with two muons using a sample of $2.4 \mathrm{fb}^{-1}$ of data. The cross section is measured as a function of the jet transverse momentum $p_{T}^{\text {jet }}$ and the jet multiplicity for jets in the kinematic region $p_{T}^{\text {jet }} \geq 30 \mathrm{GeV} / c$ and $\left|y^{\text {jet }}\right| \leq 2.1$. The transverse momentum $p_{T}$ is defined as the momentum projection in the transverse plane with respect to the colliding beams: $p_{T}=p \cos \vartheta$. $y$ indicates the rapidity, defined using the energy and the longitudinal momentum as $\frac{1}{2} \log \left(\frac{E+p_{L}}{E-p_{L}}\right)$. The measured cross sections are unfolded back to particle level using the Monte Carlo simulation and are compared to next-to-leading order (NLO) pQCD predictions including non-perturbative contributions. Fig. 1 shows the measured inclusive jet differential cross section as a function of $p_{T}^{\text {jet }}$ in the sample $Z / \gamma^{*}$ plus two or more jets compared to NLO pQCD predictions. The measurements are in good accord with the NLO predictions. 

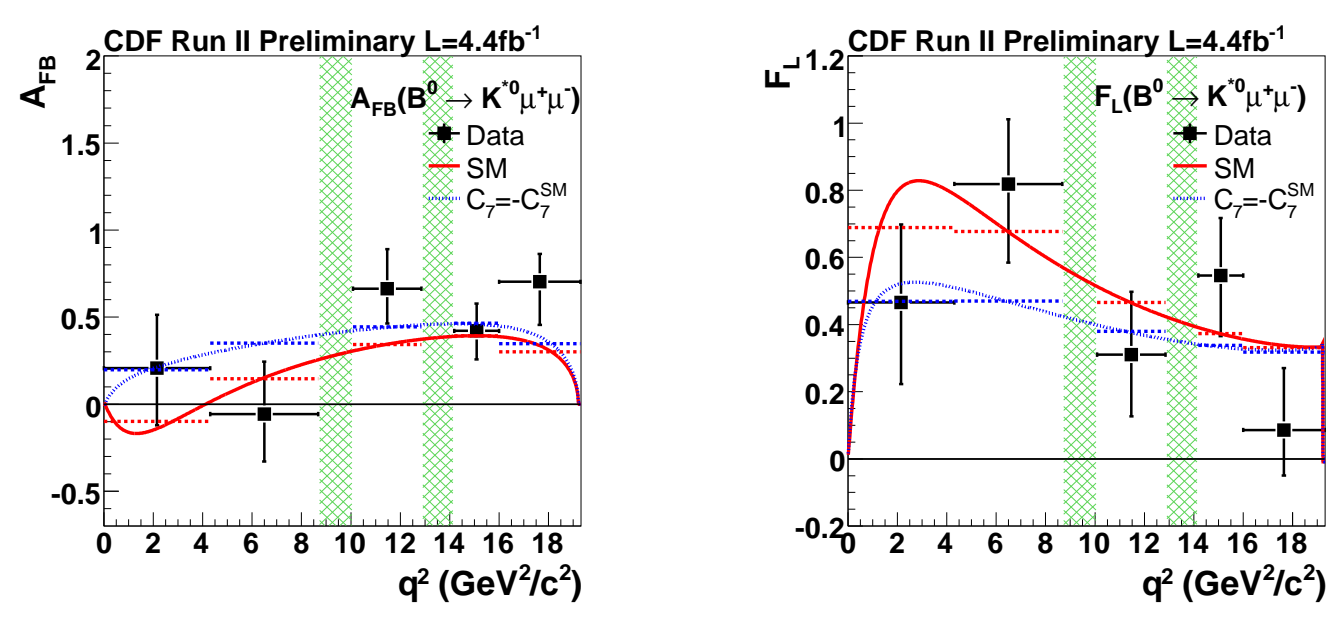

Figure 3: Muon forward-backward asymmetry $A_{F B}$ (left) and $K^{0 *}$ longitudinal polarization $F_{L}$ (right) for $B^{0} \rightarrow \mu^{+} \mu^{-} K^{0 *}$ as a function of the dimuon invariant mass $q^{2}$. The plots are described in the text.

The study of particles containing strange quarks is expected to provide valuable information on the particle production mechanism in $p \bar{p}$ collisions. For instance, if a quark-gluon plasma is formed, one of its signatures is expected to be an enhanced production of strange particles [B]. CDF has measured the inclusive differential cross section of centrally produced $(|\eta|<1) \Lambda^{0}, \Xi^{ \pm}$and $\Omega^{ \pm}$ hyperons as a function of the transverse momentum in a sample of $\sim 100$ million minimum biased events. $\Lambda^{0}, \Xi^{ \pm}$and $\Omega^{ \pm}$are $u d s, d s s$ and sss bound states, respectively. The three particles are fully reconstructed in the following decay channels, including the charge-conjugate modes: $\Lambda^{0} \rightarrow p \pi^{-}$, $\Xi^{-} \rightarrow \Lambda^{0} \pi^{-}$and $\Omega^{-} \rightarrow \Lambda^{0} K^{-}$. Fig. 2 reports the measured inclusive $p_{T}$ differential cross sections. Data points are fitted with the power law function $A p_{0}^{n} /\left(p_{T}-p_{0}\right)^{n}$, where the parameter $p_{0}$ is fixed at $1.3 \mathrm{GeV} / c$ in order to compare with the CDF Run I result on $K_{S}$ production [ [ 4 . The results of the fits for $p_{T}>2 \mathrm{GeV} / c$ are listed in Tab. 1. The production fraction of the three particles as as function of $p_{T}$ is fairly constant and drops by a factor of $\sim 7$ for each additional $s$ quark.

\subsection{Heavy flavor physics}

The abundant yield of $b$-hadrons at the Tevatron collider (the $b \bar{b}$ production cross section is $\sim 100 \mu \mathrm{b}$ ) and the exceptional quality of the detector tracking system allows CDF to pursue a heavy flavor physics program which is competitive in precision with the results from $B$-factories and complementary to them, since CDF has also access to all the $b$-hadron states. Besides studying the properties of $b$ hadrons and providing precise tests of the Standard Model, $B$ physics has the potential to give indirect evidence for new undiscovered particles that may contribute in the higher order loop-diagrams.

\begin{tabular}{lccc}
\hline \hline parameter & $\Lambda^{0}$ & $\Xi^{ \pm}$ & $\Omega^{ \pm}$ \\
\hline$A\left[\mathrm{mb} / \mathrm{GeV}^{2} c^{3}\right]$ & $170 \pm 12$ & $1.44 \pm 0.27$ & $1.35 \pm 0.60$ \\
$n$ & $8.7 \pm 0.04$ & $8.20 \pm 0.14$ & $7.90 \pm 0.32$ \\
\hline \hline
\end{tabular}

Table 1: Fit results for the inclusive differential cross sections of $\Lambda^{0}, \Xi^{ \pm}$and $\Omega^{ \pm}$for $p_{T}>2 \mathrm{GeV} / c$. 
Using a dataset of $4.3 \mathrm{fb}^{-1} \mathrm{CDF}$ has produced the currently most precise measurement of $b$-hadron lifetimes in the modes $B^{+} \rightarrow J / \psi K^{+}, B^{0} \rightarrow J / \psi K^{0 *}, B^{0} \rightarrow J / \psi K_{S}, \Lambda_{b} \rightarrow J / \psi \Lambda^{0}$. According to the spectator model the lifetimes of hadrons containing a $b$-quark and light-quark partners are determined by the weak decay of the $b$ quark [5]. The spectator model predicts equal lifetimes for $B^{+}, B^{0}$ and $\Lambda_{b}$ within a $10 \%$ correction which accounts for kinematic effects, spin interactions, Pauli interference, weak scattering and weak annihilation. The lifetimes are extracted from simultaneous unbinned maximum-likelihood fits to the reconstructed mass, the reconstructed proper decay time and the reconstructed proper decay time uncertainty. To improve the resolution on the proper decay time the $b$-hadron vertex is measured using only the $J / \psi$ tracks. The results of the fits are:

$$
\begin{aligned}
\tau_{B^{+}} & =1.639 \pm 0.009_{\text {stat }} \pm 0.009_{\text {syst }} \mathrm{ps}, \\
\tau_{B^{0}} & =1.507 \pm 0.010_{\text {stat }} \pm 0.008_{\text {syst }} \mathrm{ps}, \\
\tau_{\Lambda_{b}} & =1.537 \pm 0.045_{\text {stat }} \pm 0.014_{\text {syst }} \mathrm{ps} .
\end{aligned}
$$

The ratios of the $B^{+}$and $\Lambda_{b}$ lifetimes to the $B^{0}$ lifetime are also calculated:

$$
\begin{aligned}
& \tau_{B^{+}} / \tau_{B^{0}}=1.088 \pm 0.009_{\text {stat }} \pm 0.004_{\text {syst }}, \\
& \tau_{\Lambda_{b}} / \tau_{B^{0}}=1.020 \pm 0.030_{\text {stat }} \pm 0.008_{\text {syst }} .
\end{aligned}
$$

The theoretical predictions for the ratios are in the range 1.04-1.08 for $\tau_{B^{+}} / \tau_{B^{0}}$ and $0.83-0.93$ for $\tau_{\Lambda_{b}} / \tau_{B^{0}}$.

The $B$ meson decays $B^{+} \rightarrow K^{+} \mu^{+} \mu^{-}, B^{0} \rightarrow K^{0 *} \mu^{+} \mu^{-}$, and $B_{s}^{0} \rightarrow \phi \mu^{+} \mu^{-}$are mediated by the flavor changing neutral current transition $b \rightarrow s \mu \mu$, which is forbidden at the tree level in the Standard Model and may occur only via higher order electroweak penguin and $W$-box diagrams. New physics could enhance the rate and modify the decay distributions. Although those modes have very small branching ratios, of the order of $10^{-6}$, they are experimentally very clean. CDF has accomplished the first observation of the decay $B_{s}^{0} \rightarrow \mu^{+} \mu^{-} \phi$ with a $6.3 \sigma$ statistical significance and measured its branching ratio: $B R\left(B_{s}^{0} \rightarrow \mu^{+} \mu^{-} \phi\right)=\left(1.44 \pm 0.33_{\text {stat }} \pm 0.46_{\text {syst }}\right) \times 10^{-6}$. This represents the rarest $B_{s}^{0}$ decay observed so far. Moreover, CDF has measured the muon forwardbackward asymmetry $A_{F B}$ and the $K^{0 *}$ longitudinal polarization $F_{L}$ in the $B^{0}$ decay. In Fig. 3 the measurements (black dots) as a function of the squared dimuon mass $q^{2}=M_{\mu \mu}^{2} c^{2}$ are compared to the Standard Model prediction (red solid line). The blue dotted line shows an example of what could be the effect of new physics. The green hatched bands are the charmonium veto regions.

\subsection{Top quark physics}

The top quark provides a unique testing ground for the validity of the Standard Model. Because of its large mass it decays immediately without hadronizing and its properties are transmitted to the decay products. At the Tevatron the top quarks are predominantly produced in $t \bar{t}$ pairs via the strong interaction $q \bar{q}$ or $g g$ annihilation with a cross section of about $7 \mathrm{pb}$. In the Standard Model the top quark is expected to decay primarily into a $W$ boson and a $b$ quark according to the Cabibbo-Kobayashi-Maskawa quark mixing matrix.

It is common practice at CDF to classify the $t \bar{t}$ final state into a dilepton channel, a lepton plus jets channel and an all hadronic channel, depending on the decay modes of the two $W$ 's. The three measurements described in the following are carried out in the lepton plus jets sample. 


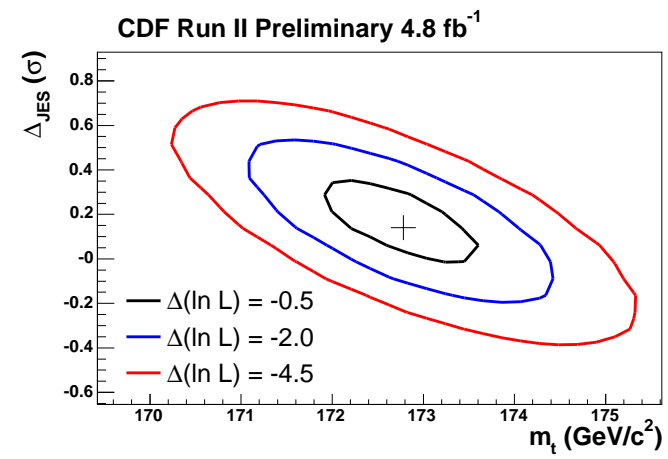

Figure 4: Likelihood maximum in the top mass and jet energy scale plane. The contours correspond to $1 \sigma, 2 \sigma$ and $3 \sigma$ confidence regions.

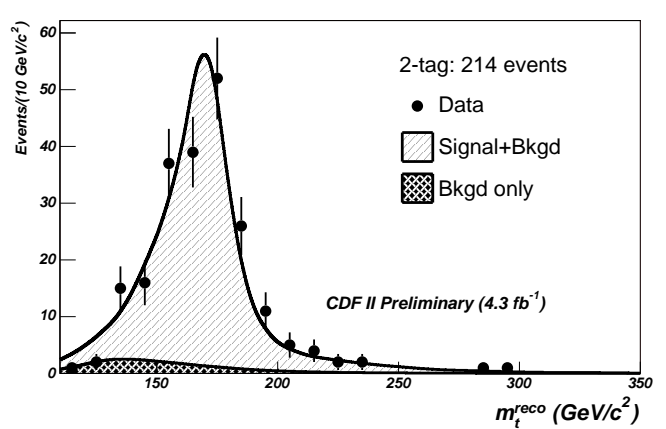

Figure 5: Reconstructed top mass overlaid with the probability density functions from the Monte Carlo sample with $\Gamma_{t}=1.5 \mathrm{GeV}$.

The top quark mass is a fundamental parameter of the Standard Model: because of its large value the top mass represents the dominant contribution in many electroweak predictions, moreover along with the $W$ mass it provides a tight constraint on the Higgs boson mass. The most recent CDF measurement of the top quark mass $m_{t}$ uses $4.8 \mathrm{fb}^{-1}$ of data. An artificial neural network is used to distinguish the signal from the background and the top mass is extracted from a maximumlikelihood fit in which the probability density function of the signal is calculated by integrating the leading-order matrix element of the $t \bar{t}$ production process. A technique to minimize the effect of the uncertainty on the jet energy measurements, the source of the largest systematic error in the measurement of the top mass, consists in determining the jet energy scale (JES) in the fit by constraining the mass of the $W$ that decays into two jets. Fig. 4 shows the likelihood maximum and the $1 \sigma, 2 \sigma$ and $3 \sigma$ contours in the plane $m_{t}-\Delta_{\mathrm{JES}}$, where $\Delta_{\mathrm{JES}}$ is a shift applied to all jet energies in units of the jet-dependent systematic uncertainty. The fit returns $m_{t}=172.8 \pm 0.7_{\text {stat }} \pm 0.6_{\mathrm{JES}} \pm$ $0.8_{\text {syst }} \mathrm{GeV} / \mathrm{c}^{2}$, a result which equals in precision the 2009 Tevatron combination of the top mass.

A determination of the decay width of the top quark permits to check the consistency of the Standard Model and to set limits on other possible decay modes like, for example, $t \rightarrow W q, t \rightarrow Z q$ or $t \rightarrow H^{+} b$. CDF has performed a measurement of the top quark decay width in $4.3 \mathrm{fb}^{-1}$ of data using a method based on templates: several Monte Carlo samples are generated with different values of the top width, for each event the top and $W$ masses are reconstructed and the top width is then extracted by comparing the two-dimensional templates to the corresponding data distribution using a maximum-likelihood method. Fig. 5 shows the distribution of the reconstructed top mass with the probability density functions from the Monte Carlo sample with $\Gamma_{t}=1.5 \mathrm{GeV}$. Since the best fit does not return a significant result, a 95\% C.L. upper limit is set on the top quark decay width: $\Gamma_{t}<7.5 \mathrm{GeV}$, which corresponds to the lower limit on the top quark lifetime $\tau_{t}>$ $8.7 \times 10^{-26} \mathrm{~s}$.

The Standard Model $t \bar{t}$ production is characterized by a well defined spin correlation between $t$ and $\bar{t}$ [6], which could be modified by new production mechanisms such as via a $Z^{\prime}$ boson or a Kaluza-Klein gluon [7]. Since the top decays weakly before hadronization, the top spin information is transferred to its decay products. CDF has reported on the observation and measurement of the $t \bar{t}$ helicity fractions and spin correlations in $4.3 \mathrm{fb}^{-1}$ of data. The opposite spin fraction $f_{0}$ is extracted 


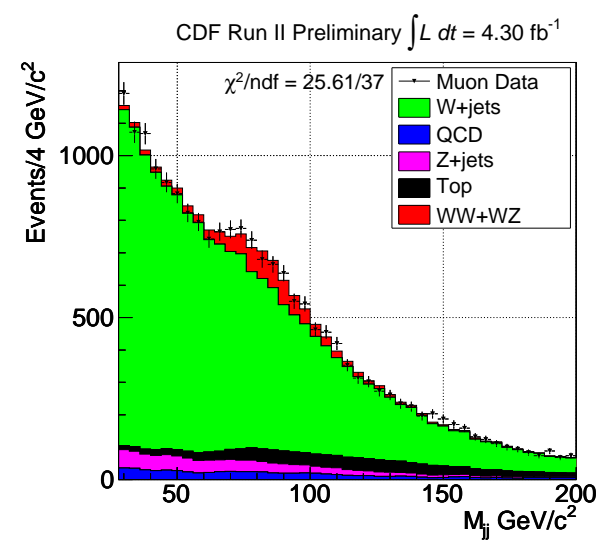

Figure 6: Reconstructed jet-jet invariant mass with the projections of the $\chi^{2}$ fit overlaid.

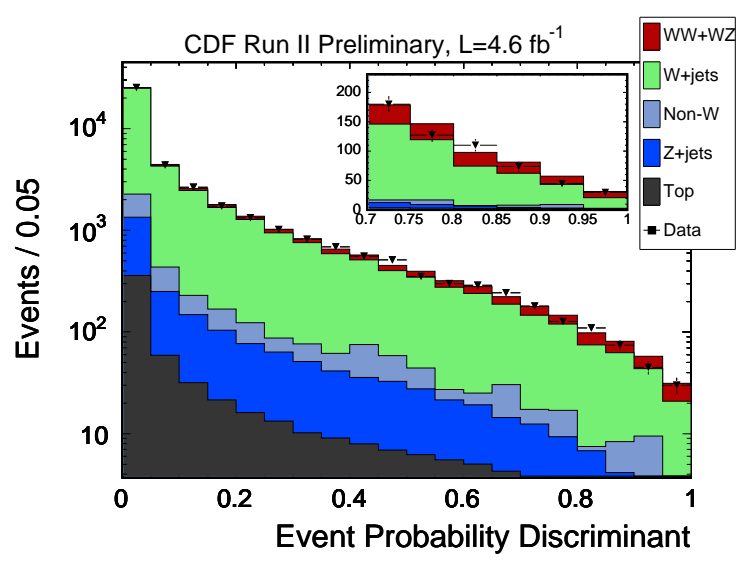

Figure 7: Event probability discriminant with the expected background superimposed.

with a simultaneous likelihood fit to two helicity bilinears, built exploiting the helicity angles in the $t \bar{t}$ rest frame of the $b$ quark and of the lepton and $d$ quark from the $W$ decays: $\cos \vartheta_{\ell} \cos \vartheta_{d}$ and $\cos \vartheta_{\ell} \cos \vartheta_{b}$. The fit returns $f_{o}=0.80 \pm 0.25_{\text {stat }} \pm 0.08_{\text {syst }}$, from which the spin correlation coefficient $\kappa$ is calculated: $\kappa=2 f_{o}-1=0.60 \pm 0.50_{\text {stat }} \pm 0.16_{\text {syst }}$.

\subsection{Electroweak physics}

At the core of the Standard Model is the electroweak sector. Understanding the production rates and the properties of the massive $W$ and $Z$ gauge bosons is of utmost importance and plays a fundamental role in the searches for new phenomena.

A major topic of the CDF electroweak program is the study of the $W W, W Z$ and $Z Z$ processes. CDF has already measured the production cross sections of all the diboson channels in the fully leptonic final states. Recently has also been observed the production of a $W$ boson in association with either another $W$ boson or a $Z$ boson in the final state with a lepton, a neutrino and two jets. This channel represents an interesting benchmark because it exhibits the same topology as a SM Higgs boson produced in association with a $W$ boson. Two independent analyses have been performed which utilize different techniques to extract the signal. The first uses a $\chi^{2}$ fit to the jet-jet reconstructed mass in $4.3 \mathrm{fb}^{-1}$, the second constructs a likelihood ratio discriminant calculating the leading-order matrix element for the signal and the main background processes in $4.6 \mathrm{fb}^{-1}$. Fig. 6 shows the projection of the fit to the reconstructed jet-jet mass, whereas Fig. 7 is the distribution of the event probability discriminant. Both observations have a statistical significance greater than $5 \sigma$. The measured cross sections are $18.1 \pm 3.3_{\text {stat }} \pm 2.5_{\text {syst }} \mathrm{pb}$ and $16.5_{-3.0}^{+3.3} \mathrm{pb}$, respectively, to be compared to the Standard Model prediction of $15.1 \pm 0.9 \mathrm{pb}$.

To test the production mechanism of the $Z / \gamma^{*}$ boson in $p \bar{p}$ collisions CDF has measured the rapidity differential cross section $d \sigma / d y$ over the full kinematical range and the total inclusive production cross section in the $e^{+} e^{-}$channel using $2.1 \mathrm{fb}^{-1}$ of data [8]. The differential cross section is then compared to the next-to-leading (NLO) and next-to-next-to-leading order perturbative QCD calculations performed with different parton distribution functions. Data turn out to be most consistent with the NLO prediction which uses the NLO CTEQ6.1M parton distribution 


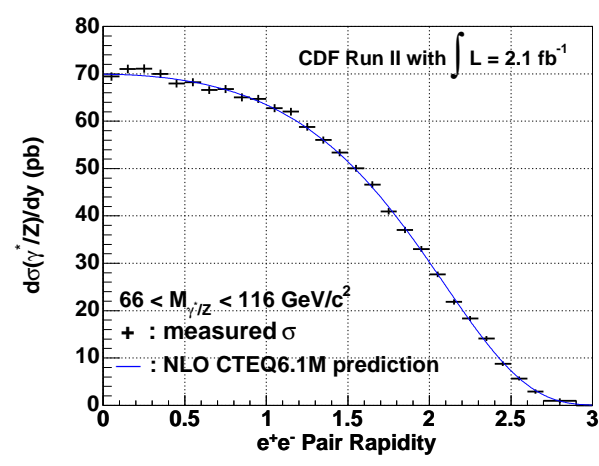

Figure 8: Measured $d \sigma / d y$ for $Z / \gamma^{*}$ (points) with the NLO prediction superimposed (solid line).

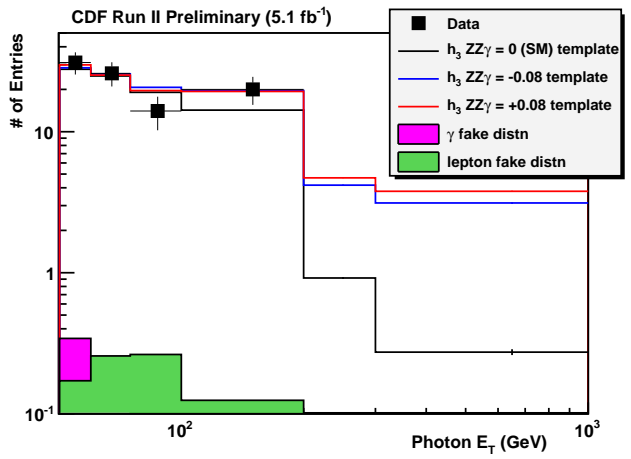

Figure 9: Transverse energy spectrum of the photon in a sample of $Z \gamma$ events.

functions [9]. Fig. 8 shows the measured differential cross section as a function of the rapidity with the theory prediction superimposed. An integration over the rapidity yields the total cross section: $\sigma_{Z / \gamma^{*}}=256.6 \pm 0.7_{\text {stat }} \pm 15.3_{\text {syst }} \mathrm{pb}$.

The study of possible $Z-\gamma$ couplings is an important test of the SM description of the gauge boson self-interactions and is potentially sensitive to physics beyond the Standard Model. In particular, the $Z \gamma$ production is directly sensitive to the triple couplings $Z Z \gamma$ and $Z \gamma \gamma$. In the Standard Model the neutral electroweak gauge boson vertices are forbidden and the only contributions to the $Z \gamma$ cross section originate from the initial and final state photon radiation. With $5.1 \mathrm{fb}^{-1}$ of data CDF has carried out a search for anomalous trilinear $Z-\gamma$ couplings in a sample of $Z$ bosons reconstructed in the $e e$ and $\mu \mu$ modes. The transverse energy spectrum of the photon, shown in Fig. 9 , is sensitive to any anomalous gauge couplings. The black solid line represents the SM prediction while the red and blue lines show two examples of non-null neutral triple gauge couplings. Using the photon spectrum 95\% C.L. upper limits are placed on the $C P$-conserving couplings listed in Tab. 2 (a definition of the couplings may be found in [11]).

\subsection{Search for the Standard Model Higgs boson}

The Higgs boson is a remnant of the mechanism which has been postulated to account for the electroweak symmetry breaking and the generation of particle masses. It represents the last piece of the Standard Model which is awaiting an experimental confirmation. Although the Higgs boson mass cannot be predicted by theory, indications on its value are provided by the direct searches conducted at LEP, which excluded the mass region $m_{H}<114.4$ at 95\% C.L. [12], by indirect constraints from the $W$ and top masses and by precision global electroweak fits, which seem to

\begin{tabular}{lcccc}
\hline \hline & $h_{3}^{\gamma}$ & $h_{4}^{\gamma}$ & $h_{3}^{Z}$ & $h_{4}^{Z}$ \\
\hline expected limit & $0.042_{-0.008}^{+0.012}$ & $0.0019_{-0.0002}^{+0.0005}$ & $0.043_{-0.009}^{+0.013}$ & $0.0020_{-0.0003}^{+0.0005}$ \\
measured limit & {$[-0.037,0.038]$} & {$[-0.0017,0.0017]$} & {$[-0.038,0.040]$} & {$[-0.0017,0.0017]$} \\
\hline \hline
\end{tabular}

Table 2: Expected and measured 95\% C.L. limits on anomalous neutral gauge boson self-couplings. 


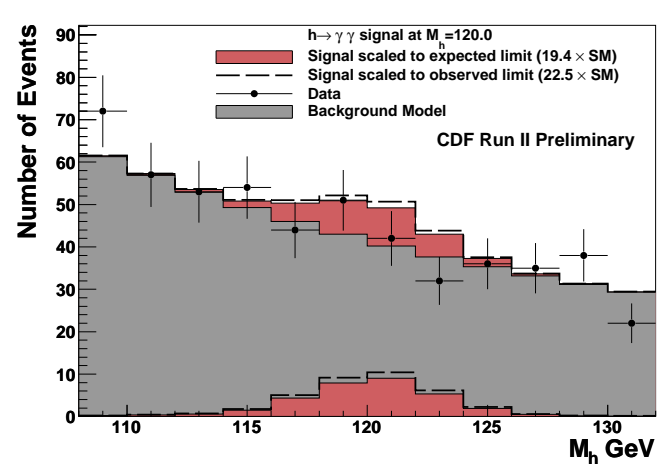

Figure 10: Diphoton invariant mass distribution with the expected Standard Model backgrounds and an hypothetical Higgs signal at $120 \mathrm{GeV} / c^{2}$.

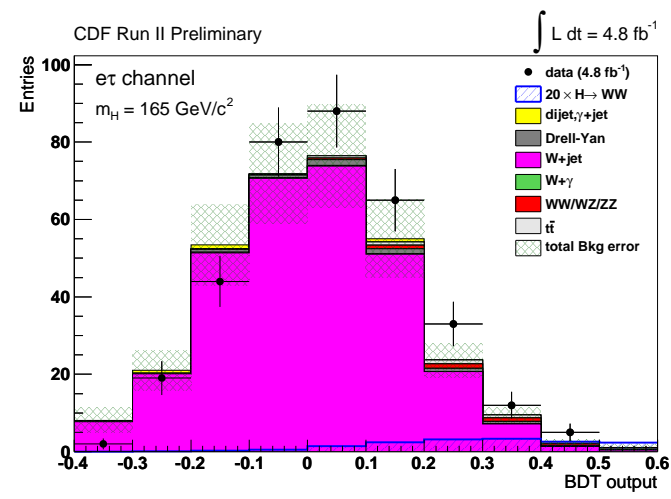

Figure 11: Distribution of the Boosted Decision Tree score in the electron-tau sample for a Higgs boson mass of $165 \mathrm{GeV} / c^{2}$.

prefer a relatively light Higgs [13]: the preferred value for $m_{H}$ is at $87_{-26}^{+35} \mathrm{GeV} / c^{2}$, while $m_{H}<157$ at $95 \%$ C.L.

At a $p \bar{p}$ center of mass energy of $1.96 \mathrm{TeV}$ the Higgs boson is produced predominantly via a gluon-gluon fusion process through a quark loop, where the main contribution is given by the massive top quark [14]. The cross section decreases from $\sim 1.5$ to $\sim 0.2 \mathrm{pb}$ as the Higgs boson mass varies between 100 and $200 \mathrm{GeV} / \mathrm{c}^{2}$. The rates of the electroweak production mechanisms in association with a $W$ or $Z$ boson or via vector boson fusion are about an order of magnitude lower. $\mathrm{CDF}$ is pursuing a direct search for the SM Higgs boson over the mass range $100-200 \mathrm{GeV} / \mathrm{c}^{2}$. The search strategy is driven by the dominant decay modes of the Higgs boson.

For masses below $\sim 135 \mathrm{GeV} / c^{2}$ the Higgs decays predominantly into a $b \bar{b}$ pair. An inclusive search in this mass range would be spoiled by the overwhelming multijet background, therefore the channels with an associated $W$ or $Z$ boson are in general more convenient. The most promising discovery channel is represented by $W H \rightarrow \ell v_{\ell} b \bar{b}$. CDF has recently updated the search with a dataset of $4.8 \mathrm{fb}^{-1}$. The analysis requires high transverse missing energy, a high- $p_{T}$ lepton and two $b$-tagged jets. The transverse missing energy is defined as an imbalance of the total energy deposited in the calorimeter and accounts for the neutrino which escapes detection. The $b$-jet identification (tagging) exploits the relatively longer lifetime of $b$ quarks either looking for secondary displaced vertices inside the jets or using the impact parameters of the tracks belonging to the jet. An event probability, based on the leading-order matrix element calculation, is used to discriminate the signal from the Standard Model backgrounds. The measured 95\% C.L. upper limits on the production cross section times the branching ratio of the Higgs boson range from 2.9 to 31.4 times the SM cross section for masses between 100 and $150 \mathrm{GeV} / c^{2}$. The expected 95\% C.L. limits are between 2.9 and 29.8 .

Another analysis in the low mass range uses $2.3 \mathrm{fb}^{-1}$ of data to search for a Higgs boson associated to one or more jets and decaying to two tau leptons. Most of the signal events with this signature are produced via the gluon-gluon fusion and the vector boson fusion processes. The $H \rightarrow \tau \tau$ branching fraction is approximately one tenth of the $b \bar{b}$ mode. A multivariate technique based on a Boosted Decision Tree (BDT) is used to separate the signal from the backgrounds. The 


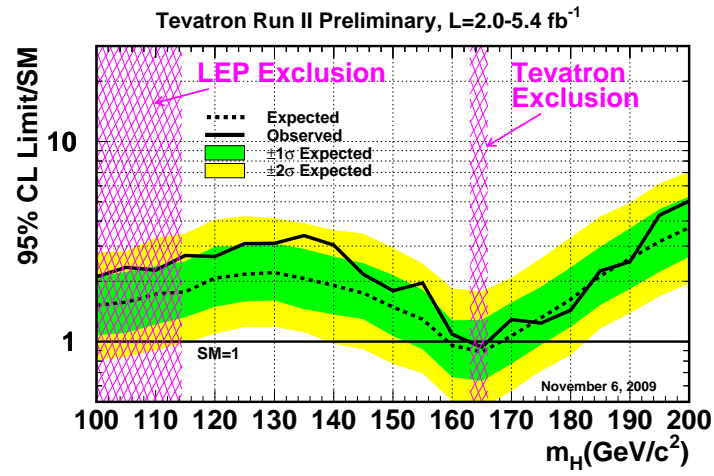

Figure 12: November 2009 Tevatron combination of the observed and expected $95 \%$ C.L. upper limits on the Higgs boson production cross section.

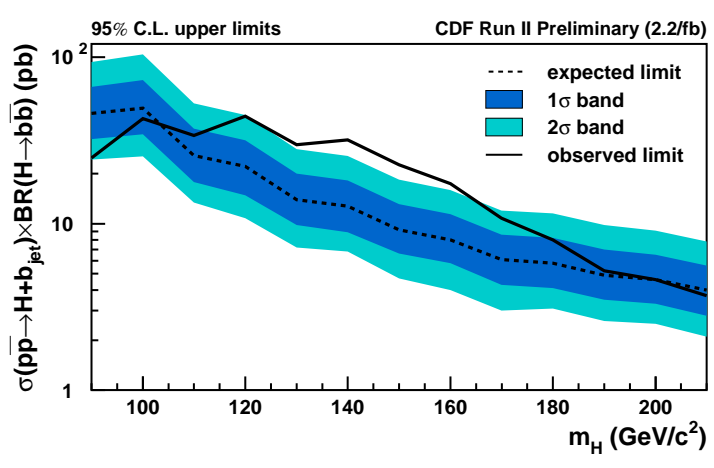

Figure 13: $95 \%$ C.L. upper limits on the production cross section times the branching ratio for a light neutral Higgs boson associated to a $b$-quark.

CDF sensitivity to this channel is highest at a test mass of $120 \mathrm{GeV} / c^{2}$, for which value an upper limit of 23.4 times the Standard Model cross section is expected at 95\% C.L. The corresponding observed $95 \%$ C.L. upper limit is 27.2 .

The Higgs decay to two photons provides the cleanest experimental signature, but suffers from a very low branching ratio in the Standard Model, $\sim 0.2 \%$ for $m_{H}=120 \mathrm{GeV} / \mathrm{c}^{2}$. Nonetheless, it is worth investigating because the branching ratio is enhanced in some scenarios beyond the Standard Model [15]. The diphoton invariant mass is a very good discriminant, since its resolution is only a few $\mathrm{GeV} / \mathrm{c}^{2}$. The distribution is shown in Fig. 10. In a $5.4 \mathrm{fb}^{-1}$ dataset the $95 \%$ C.L. upper limits on the production cross section times the branching fraction relative to the SM expectation range from 18.7 to 40.0 for Higgs masses between 100 and $150 \mathrm{GeV} / c^{2}$. The sensitivity is in the range between 19.4 and 38.6.

For masses higher than $135 \mathrm{GeV} / c^{2}$ the decay to $W W$ is dominant. In this case the final states with leptons (i.e. muons and electrons) offer very clean experimental signatures with relatively low backgrounds. To better characterize the backgrounds and improve the signal to background separation, the search is divided into sub-channels which are analysed separately and then combined: two opposite-sign leptons with zero, one or more than two jets, two opposite sign-leptons with an invariant mass lower than $16 \mathrm{GeV} / c^{2}$, two same-sign leptons, three leptons, a lepton plus a tau decaying to hadrons. Multivariate methods are utilized to discriminate signal and background. Fig. 11 shows an example of the BDT discriminant used in the analysis with taus. The highest sensitivity is achieved at $165 \mathrm{GeV} / c^{2}$, where a 95\% C.L. upper limit of 1.02 times the SM production cross section is expected. For the same mass CDF measures an upper limit of $1.11 \times \sigma_{\mathrm{SM}}$.

In order to increase the sensitivity to the production of the SM Higgs boson, the CDF and DØ experiments combine regularly the results of their searches. Fig. 12 shows the November 2009 Tevatron combination. The graph reports the expected and observed 95\% C.L. upper limits on the ratios to the SM cross section as functions of the Higgs boson mass. The limits are expressed as a multiples of the SM prediction for each test mass. The bands indicate the $68 \%$ and $95 \%$ probability regions where the limits can fluctuate in the absence of a signal. At $115 \mathrm{GeV} / c^{2}$ the sensitivity is 1.8 times the SM cross section, whereas the observed upper limit is 2.7. In the high mass region the $\mathrm{CDF}$ and $\mathrm{D} \varnothing$ exclude the masses in the range between 163 and $166 \mathrm{GeV} / \mathrm{c}^{2}$ at 95\% C.L. 


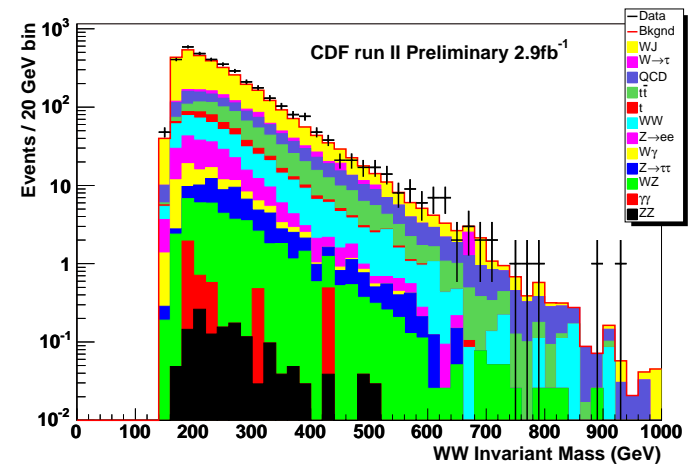

Figure 14: Reconstructed $W W$ mass in data (black dots) with the expected Standard Model backgrounds superimposed (solid histograms).

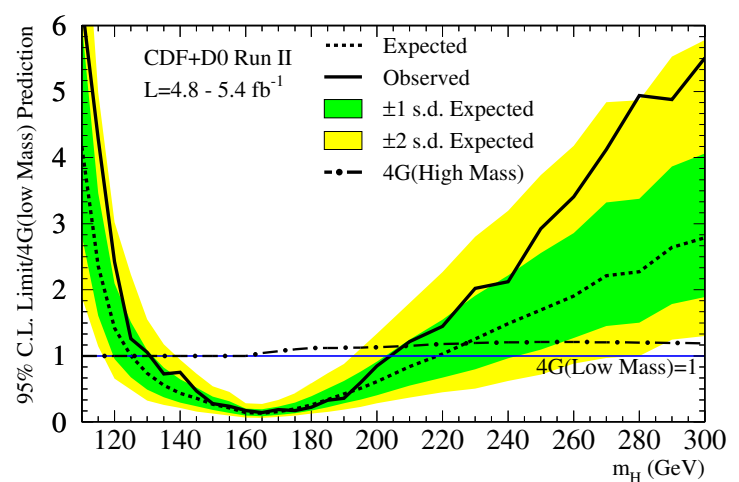

Figure 15: Ratio of the CDF-D $\varnothing$ expected and observed $95 \%$ C.L. upper limits on $\sigma_{H}$ to the theoretical predictions from a fourth-generation model.

\subsection{Beyond the Standard Model Searches}

Besides trying to unhinge the Standard Model with high precision measurements which could reveal inconsistencies in its predictions, $\mathrm{CDF}$ is also carrying out an extensive direct search for exotic processes that would not fit in the Standard Model framework.

Supersymmetric extensions of the Standard Model in general present a richer Higgs sector. In models with large values of $\tan \beta$, defined as the ratio of the Higgs couplings to down-type versus up-type quarks, the production of light Higgs bosons in association with $b$-quarks can be significantly enhanced [16]. In this context, $\mathrm{CDF}$ has completed a search for a neutral Higgs boson decaying into a $b \bar{b}$ pair and produced in association with another $b$ quark in $2.2 \mathrm{fb}^{-1}$ of data. The Higgs signal is expected to appear as an enhancement in the mass distribution of the two most energetic jets. Fig. 13 shows the 95\% C.L. upper limits on the production cross section times the branching ratio for thirteen test masses.

New physics could reveal themselves as a high-mass resonance decaying into a pair of weak gauge bosons. CDF has performed a searches for a $W W$ or $W Z$ resonance in a $2.2 \mathrm{fb}^{-1}$ sample [10]. Fig. 14 shows the distribution of the $W W$ reconstructed mass with the expected SM backgrounds superimposed. Since no excess is observed above the SM backgrounds, 95\% C.L. limits are set on the production cross section for three resonance hypotheses: a Randall-Sundrum graviton $G^{*}$, a $W^{\prime}$ and a $Z^{\prime}$ boson. By comparing the limits with the predicted cross sections, mass exclusion regions are determined for each resonance hypothesis. The results are summarized in Tab. 3 .

One of the possible extensions of the SM predicts the existence of a hypothetical fourth generation of fermions with masses much larger than the first three generations [17]. In such a scenario the Higgs boson coupling to the gluon is enhanced by the contributions in the quark loop from the new massive particles and, accordingly, the $g g \rightarrow H$ production rate increases by a factor of

\begin{tabular}{lccc}
\hline \hline & $G^{*}$ & $Z^{\prime}$ & $W^{\prime}$ \\
\hline Expected mass exclusion $\left[\mathrm{GeV} / c^{2}\right]$ & $<632$ & $257-630$ & $381-421$ \\
Data mass exclusion $\left[\mathrm{GeV} / c^{2}\right]$ & $<607$ & $247-544$ & $285-516$ \\
\hline \hline
\end{tabular}

Table 3: Excluded mass ranges for the Randall-Sundrum graviton $G^{*}$ and the $Z^{\prime}$ and $W^{\prime}$ bosons. 
$\sim 9$. While at low masses the Higgs partial decay width to $g g$ is enhanced by the same amount, the $H \rightarrow W W$ channel still dominates for masses $m_{H}>135 \mathrm{GeV} / c^{2}$. Limits on the gluon fusion production cross section provide stringent constraints on fourth-generation models. The CDF and DØ Collaborations have released a combined result [18] which, by comparing the limits on the Higgs production cross section with theoretical predictions from models with a massive fourth generation of fermions, excludes at 95\% C.L. a Higgs boson mass between 131 and $204 \mathrm{GeV} / c^{2}$. Fig. 15 shows the CDF and $\mathrm{D} \emptyset$ combined expected and observed limits on the Higgs production cross section.

\section{Conclusion}

The Tevatron is providing the CDF experiment with large datasets to be analysed: $9 \mathrm{fb}^{-1}$ have been delivered up to now and $12 \mathrm{fb}^{-1}$ are expected by the end of 2011 . Thanks to the detailed understanding of the CDF detector and the high sophistication of the analyses, the precision of CDF measurements is scaling better than the mere statistical factor given by the luminosity increase, allowing an unprecedented sensitivity to processes with cross sections of fractions of a picobarn. The siege of the Tevatron to the Standard Model continues relentless.

\section{References}

[1] D. Acosta et al. [The CDF Collaboration], Phys. Rev. D71, 032001 (2005).

[2] D.J. Gross and F. Wilczek, Phys. Rev. D8, 3633 (1973).

[3] L. McLerran, Rev. Mod. Phys. 58, 1021 (1986).

[4] F. Abe et al. [The CDF Collaboration], Phys. Rev. D40, 3791 (1989).

[5] C. Tarantino, Eur. Phys. J. C33, S895 (2004); F. Gabbiani, A. Onishchenko, and A. Petrov, Phys. Rev.D68, 114006 (2003) and; F. Gabbiani, A. Onischenko and A. Petrov, Phys. Rev. D70 094031 (2004); I.I. Bigi et al., in B Decays, edited by S. Stone, World Scientific, Singapore (1994).

[6] T. Stelzer and S. Willenbrock, Phys. Lett. B374, 169 (1996).

[7] G.L. Kane, G.A. Ladinsky and C.-P. Yuan, Phys. Rev. D45, 124 (1992); M. Arai, N. Okada, K. Smolek and V. Simak, Phys. Rev. D75, 095008 (2007).

[8] T. Aaltonen et al. [The CDF Collaboration], arXiv:0908.3914 [hep-ex].

[9] D. Stump et al., JHEP 10, 046 (2003).

[10] T. Aaltonen et al. [The CDF Collaboration], Phys. Rev. Lett. 104, 241801 (2010).

[11] G.J. Gounaris, J. Layssac and F.M. Renard, Phys. Rev. D 62, 073013 (2000).

[12] R. Barate et al [The ALEPH, DELPHI, L3 and OPAL Collaborations], Phys. Lett. B565 61 (2003).

[13] J. Alcaraz et al. [The LEP Electroweak Working Group], arXiv:0911.2604 [hep-ex].

[14] U. Aglietti et al. [The TeV4LHC Working Group], arXiv:hep-ph/0612172v2.

[15] S. Mrenna and J. Wells, Phys. Rev. D63, 015006 (2000).

[16] M. Carena, S. Heinemeyer, C.E.M. Wagner and G. Weiglein, arXiv:hep-ph/0511023v1.

[17] B. Holdom, W.S. Hou, T. Hurth, M.L. Mangano, S. Sultansoy, G. Unel, arXiv:0904.4698v2 [hep-ph].

[18] T. Aaltonen et al. [The CDF and DØ Collaborations], arXiv:1005.3216v2 [hep-ex]. 